\title{
Addition and correction
}

\section{Efficient Synthesis of the Indoloazocine Framework via Intramolecular Alkyne Carbocyclization}

\author{
Pavel A. Donets, ${ }^{\dagger}$ Kristof Van Hecke, ${ }^{\ddagger}$ Luc Van Meervelt, ${ }^{\ddagger}$ \\ and Erik V. Van der Eycken ${ }^{*, \dagger}$ \\ ${ }^{\dagger}$ Laboratory for Organic \& Microwave-Assisted Chemistry (LOMAC) \\ and ${ }^{\ddagger}$ Biomolecular Architecture, Department of Chemistry, Katholieke \\ Universiteit Leuven, Celestijnenlaan 200F, B-3001 Leuven, Belgium \\ erik.vandereycken@chem.kuleuven.be
}

\section{Supporting Information}

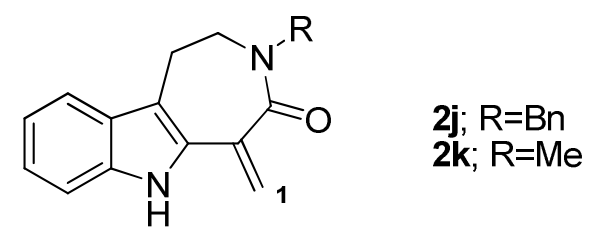

3-benzyl-5-methylene-2,3,5,6-tetrahydroazepino[4,5-b]indol-4(1H)-one (2j).

${ }^{1} \mathrm{H}$ NMR $\left(\mathrm{CDCl}_{3}, \mathbf{3 0 0} \mathbf{M H z}\right): \delta 9.00$ (bs, $\left.1 \mathrm{H}\right), 7.47-7.26(\mathrm{~m}, 7 \mathrm{H}), 7.24-7.15(\mathrm{~m}$, $1 \mathrm{H}), 7.13-7.03(\mathrm{~m}, 1 \mathrm{H}), 5.94(\mathrm{~s}, 1 \mathrm{H}), 5.75(\mathrm{~s}, 1 \mathrm{H}) 4.78(\mathrm{~s}, 2 \mathrm{H}), 3.78-3.60(\mathrm{~m}, 2 \mathrm{H})$, 2.90-2.76 (m, 2H); ${ }^{13} \mathbf{C}$ NMR $\left(\mathrm{CDCl}_{3}, 75 \mathrm{MHz}\right): \delta 170.4,138.3,137.4,136.3$, 129.0, 128.7, 128.3, 128.2, 127.6, 123.2, 120.6, 119.7, 118.6, 113.1, 111.3, 50.6, 47.0, 24.5; ${ }^{13} \mathrm{C}$ DEPT-135 NMR ( $\left.\mathrm{CDCl}_{3}, 100 \mathrm{MHz}\right): \delta$ 128.8, 128.2, 127.6, 123.2, $120.6\left(\mathrm{C}^{1}\right), 119.7,118.6,111.3,50.6\left(\mathrm{CH}_{2}\right), 47.0\left(\mathrm{CH}_{2}\right), 24.5\left(\mathrm{CH}_{2}\right)$.

3-methyl-5-methylene-2,3,5,6-tetrahydroazepino[4,5-b]indol-4(1H)-one (2k).

${ }^{1} \mathrm{H}$ NMR $\left(\mathrm{CDCl}_{3}, 400 \mathrm{MHz}\right): \delta 8.87(\mathrm{bs}, 1 \mathrm{H}), 7.47(\mathrm{~d}, 1 \mathrm{H}, \mathrm{J}=7.9 \mathrm{~Hz}), 7.39(\mathrm{~d}, 1 \mathrm{H}$, $\mathrm{J}=7.9 \mathrm{~Hz}), 7.25-7.17(\mathrm{~m}, 1 \mathrm{H}), 7.15-7.05(\mathrm{~m}, 1 \mathrm{H}), 5.84(\mathrm{~s}, 1 \mathrm{H}), 5.68(\mathrm{~s}, 1 \mathrm{H})$,

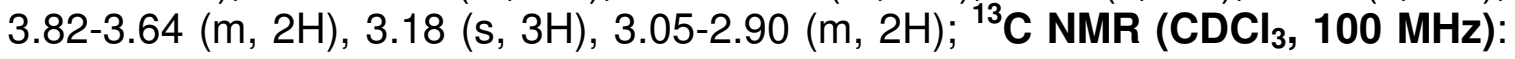
$\delta 170.1,138.3,136.2,129.1,128.3,123.2,120.0,119.8,118.6,113.1,111.2$, 49.5, 34.8, 24.0; ${ }^{13} \mathrm{C}$ DEPT-135 NMR (CDCl $\left.3,100 \mathrm{MHz}\right): \delta$ 123.2, $120.1\left(\mathrm{C}^{1}\right)$, 119.8, 118.6, 111.2, $49.5\left(\mathrm{CH}_{2}\right), 34.9,24.1\left(\mathrm{CH}_{2}\right)$. 


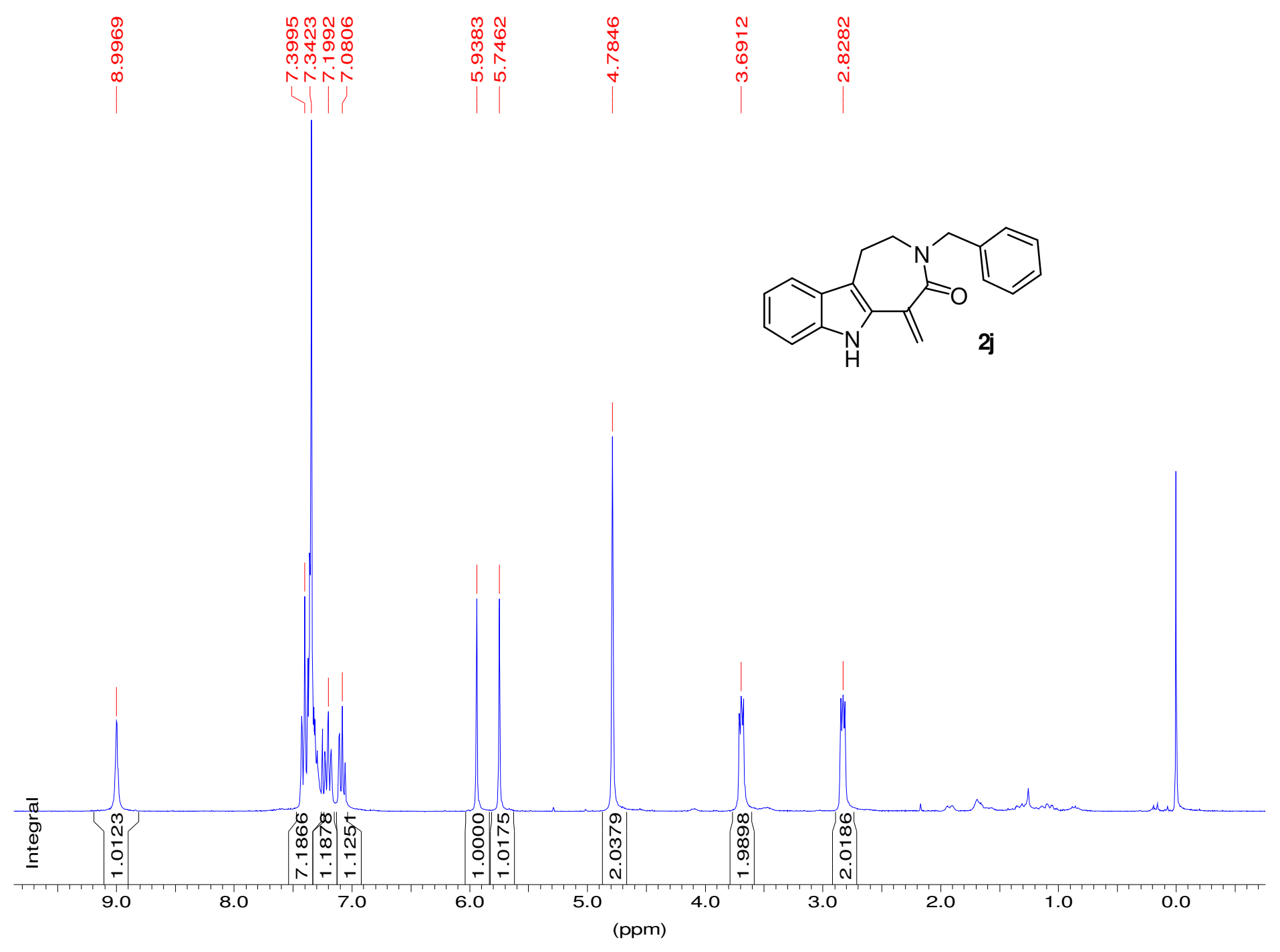




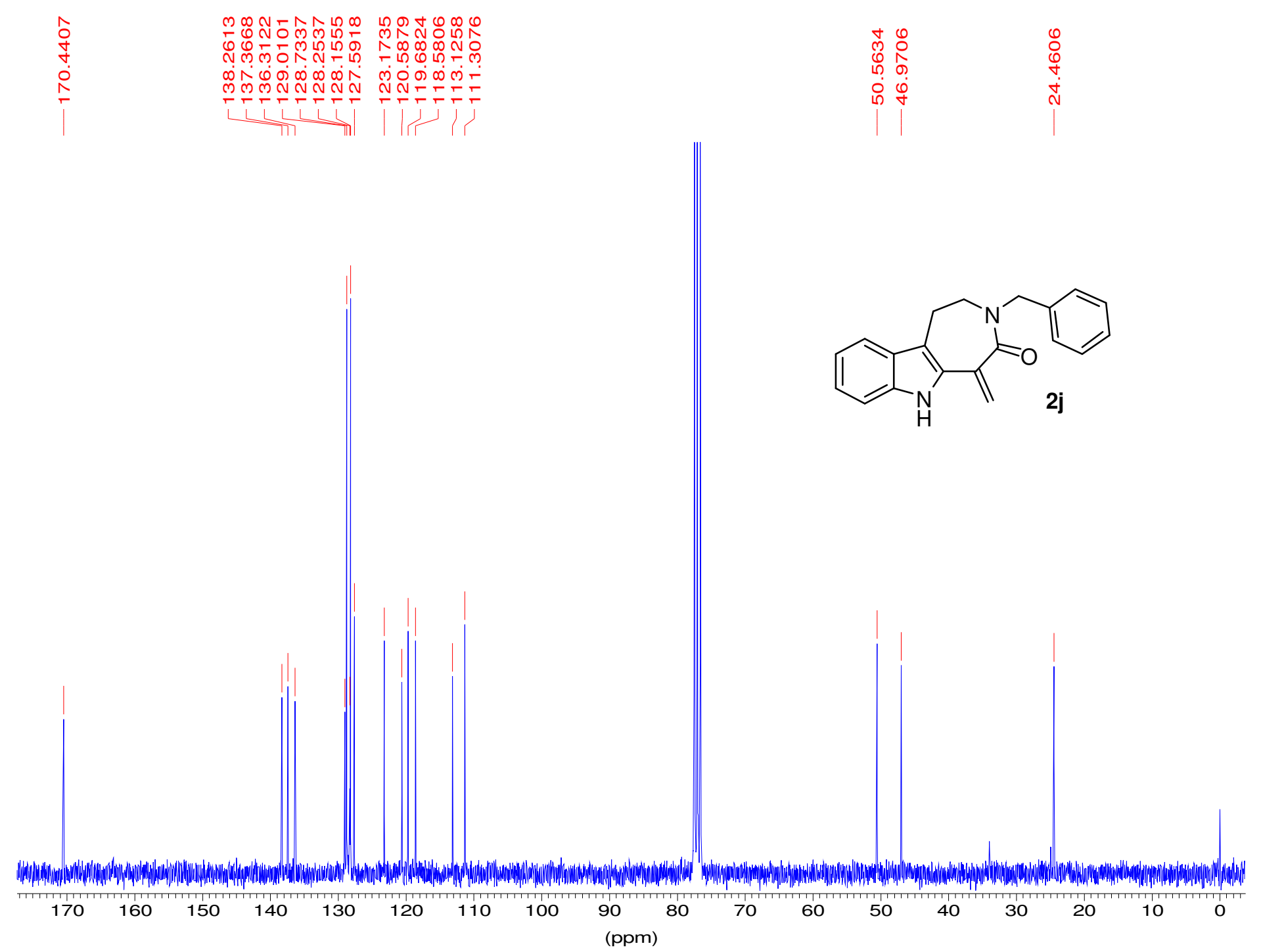




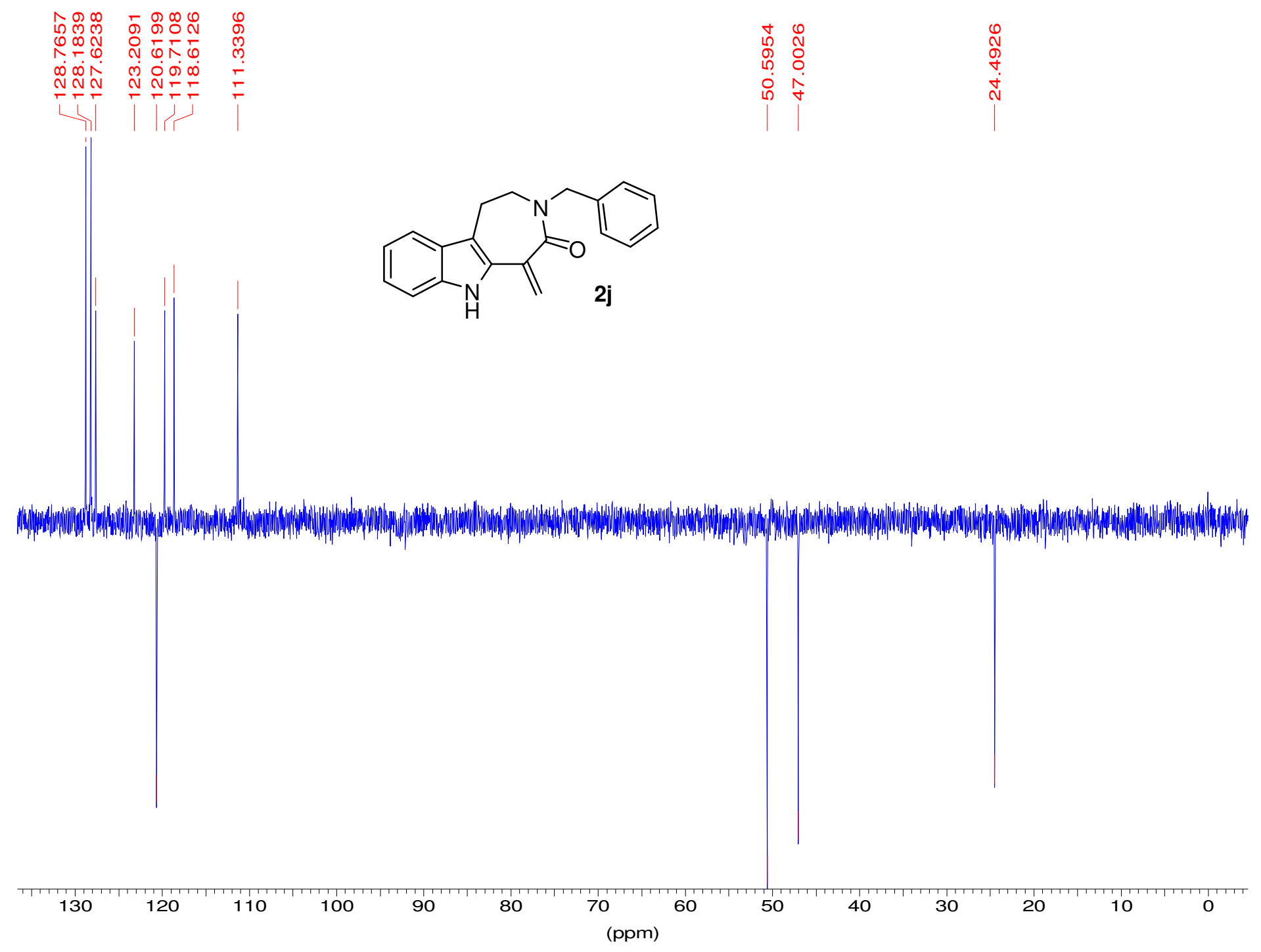




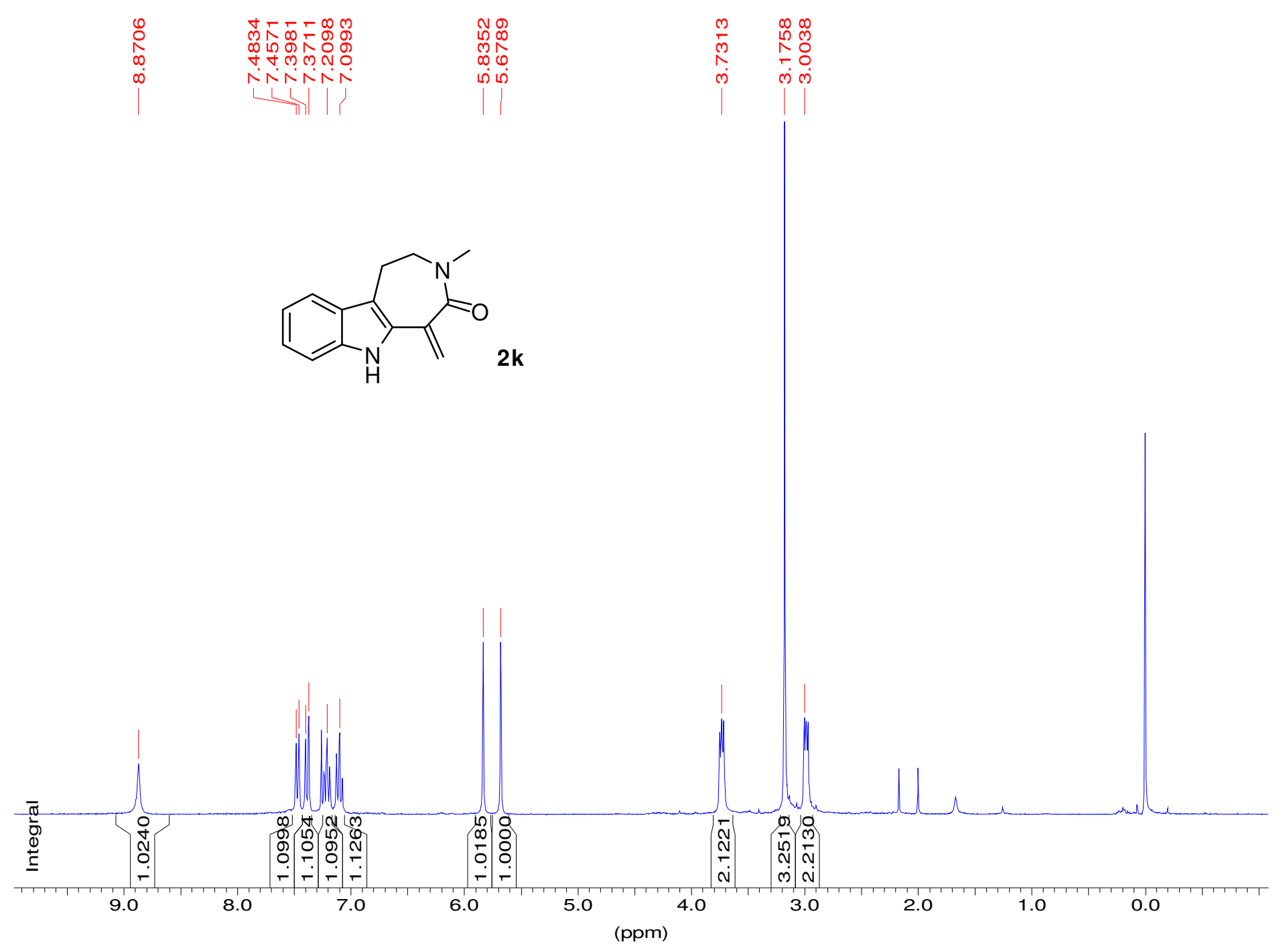




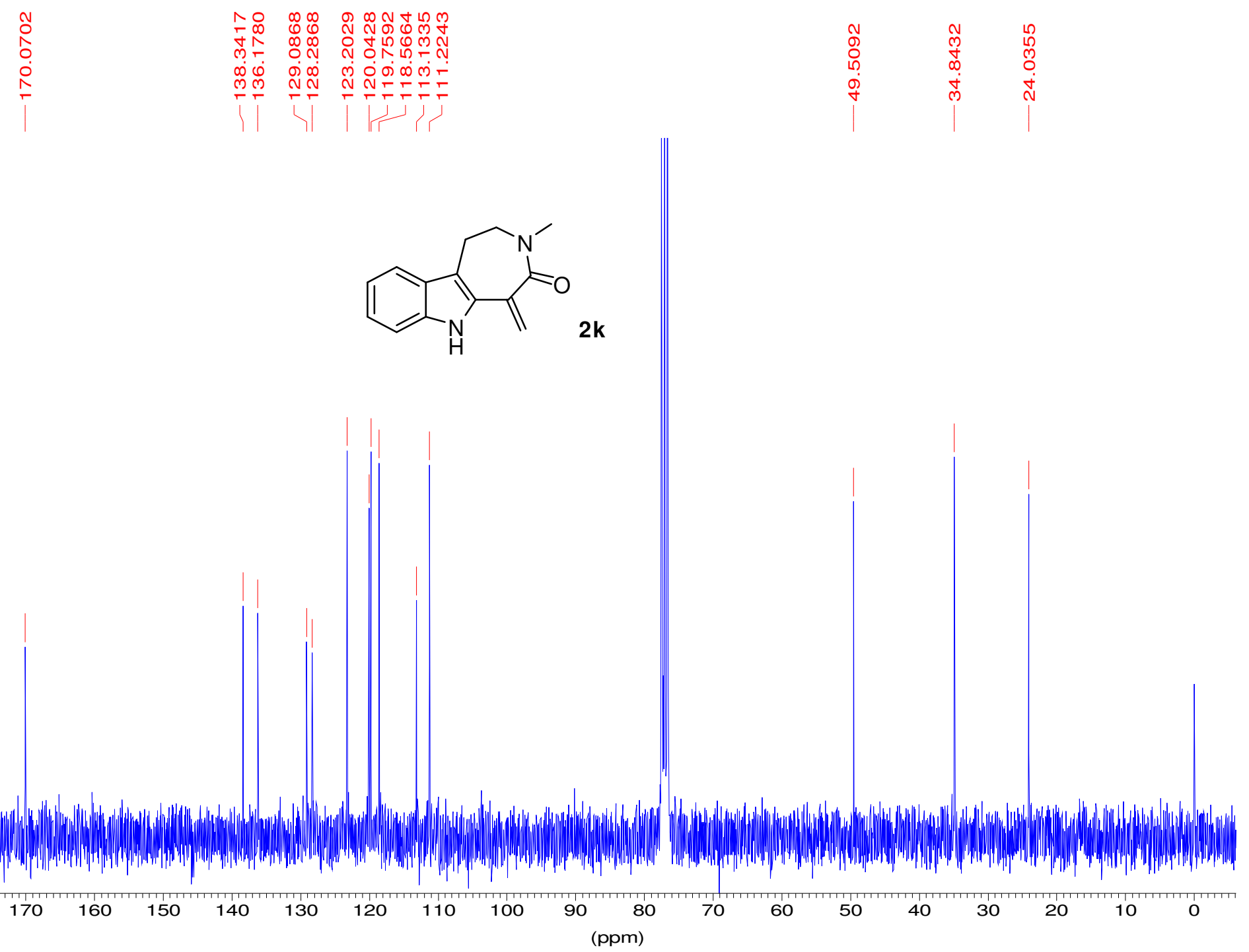




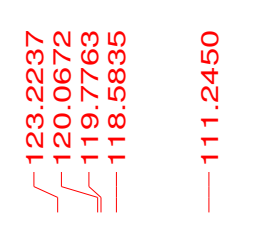

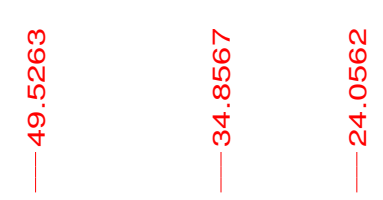

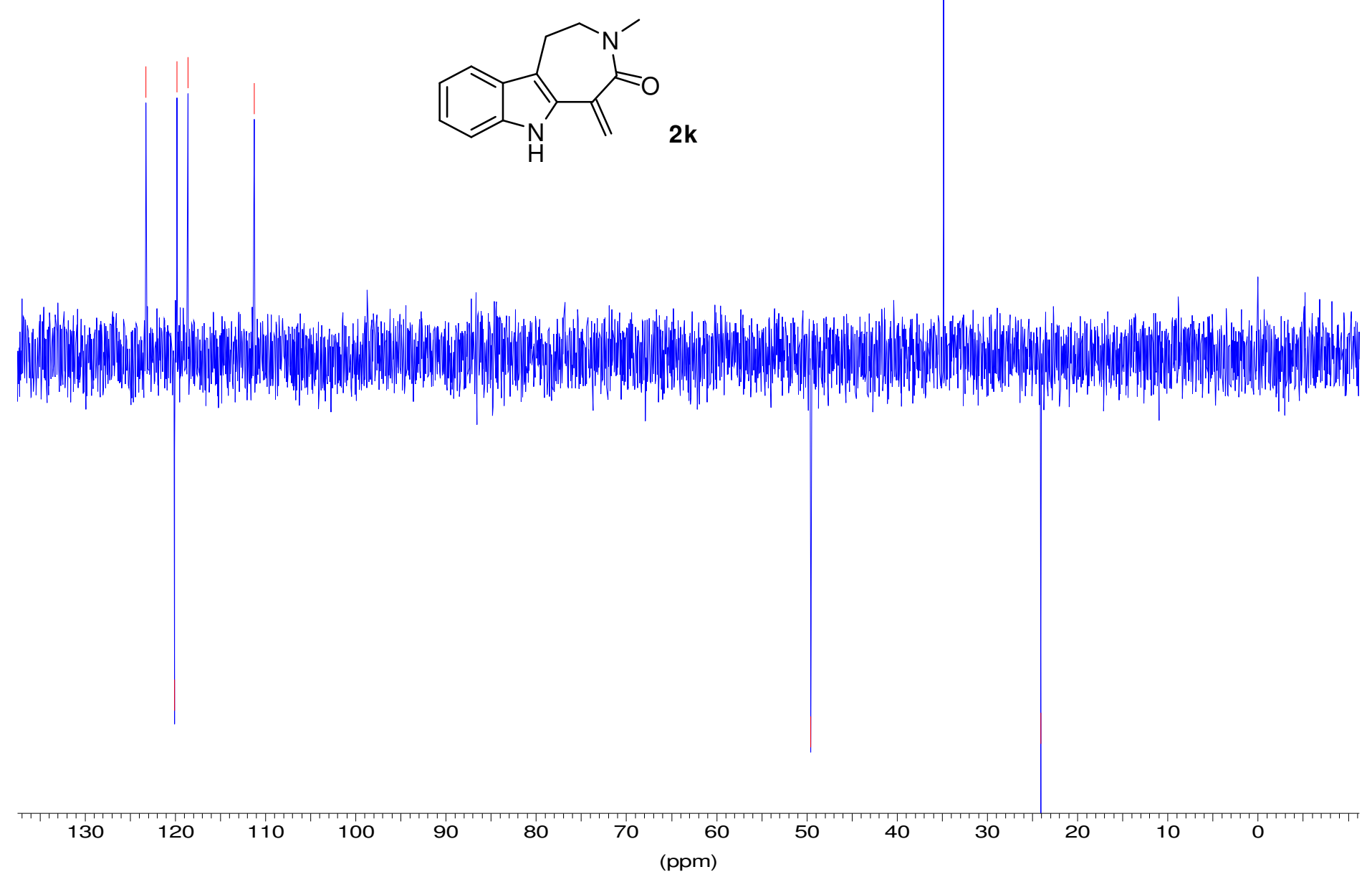

